\title{
Is it reasonable for the same city to advocate a policy of reducing heating temperature by the same amount?
}

\author{
Shiqian Wang ${ }^{1}$, Enshen Long ${ }^{1,2, *}$ and Ning $\mathrm{Mao}^{2}$ \\ ${ }^{1}$ MOE Key Laboratory of Deep Earth Science and Engineering, College of Architecture and Environment, Sichuan University, Chengdu \\ 610065, China \\ ${ }^{2}$ MOE Key Laboratory of Deep Earth Science and Engineering, Institute for Disaster Management and Reconstruction, Sichuan \\ University, Chengdu 610065, China
}

\begin{abstract}
Reducing heating temperature is a universally recognized energy saving measure. However, the same city has buildings completed in different years and with different functional USES at the same time. It is an important issue worth discussing whether it is reasonable for the government to advocate reducing heating temperature by the same range. Based on DOE medium-sized office building as the object, using the Beijing meteorological database system, the thermal characteristics of four envelopment structures representing different completion years, the annual heating energy saving and relative energy saving ratio when the set indoor heating temperature is reduced by the same range are studied. The results show that the worse the thermal performance of the envelope is, the greater the annual heating energy is when the heating setting temperature drops by the same extent. It indicates that the older the building is, the better heating energy saving effect and more heating cost can be achieved by advocating lowering the heating setting temperature of the same range. For the annual relative energy saving ratio, the older buildings with worse envelope structure are slightly higher, while the new buildings with better envelope structure are slightly lower, but they are similar on the whole. It is proved that the policy of reducing heating temperature by the same range in the same city is reasonable for achieving similar energy saving ratio.
\end{abstract}

\section{Introduction}

Energy consumption in public buildings accounts for $38 \%$ of the country's total building energy consumption. The total area of severe cold and cold areas in China is very large, accounting for about $70 \%$ of the total land area. The Chinese system of building energy efficiency standards focuses on the goal of achieving the same energy efficiency rate across the country. For public buildings, the Public Building Energy Conservation Design Standard issued in 2005 was revised and improved in 2015 [1], in which relevant provisions were made on the thermal parameters of envelope structure and the setting of building internal disturbance for different functional uses, and the requirement for energy conservation rate was increased to $65 \%$.In 2019, the Technical Standard for Nearly-zero Energy Consumption Building [2] puts forward the concepts of ultra-low energy consumption building and nearly-zero energy consumption building, and stipulates that the energy consumption levels of public buildings should be reduced by more than $50 \%$ and $60 \% \sim 75 \%$, respectively, compared with international standards [1]. It can be seen that due to the high frequency of revision of Chinese standards, in any city, there are all kinds of buildings with different envelope performance in different completion years. In terms of building energy conservation management, the government often has to introduce some guiding policies for energy conservation, such as no more than $20^{\circ} \mathrm{C}$ in winter and no less than $26{ }^{\circ} \mathrm{C}$ in summer, so as to better achieve the goal of energy conservation rate [3]. For all kinds of buildings coexisting with different functions and different completion years in the same city, what is the commonness and personality of the policy that the administrative regulation reduces the indoor heating temperature by the same range for the absolute energy saving effect and relative energy saving rate of buildings in different completion years? And is the policy reasonable? This is an important issue that scholars at home and abroad do not study much.

In terms of building energy conservation, heat transfer loss of envelope accounted for $70 \% \sim 80 \%$ of the total building loss. It can be seen that for buildings in cold and severe cold regions, reducing heat transfer loss of envelope can achieve a more prominent energy-saving effect. Nuria Casquero-Modrego et al. [4] pointed out that most of the energy consumption of heating and air conditioning in buildings comes from the heat transfer of the envelope. The author by monitoring building heating energy consumption, found in the case of indoor temperature constant, outer wall heat transfer coefficient from $1.89 \mathrm{~W} /\left(\mathrm{m}^{2} \cdot \mathrm{K}\right)$ to $0.489 \mathrm{~W} /\left(\mathrm{m}^{2} \cdot \mathrm{K}\right)$ and heat transfer coefficient of the roof from $0.625 \mathrm{~W} /\left(\mathrm{m}^{2} \cdot \mathrm{K}\right)$ to 0.308

\footnotetext{
*Corresponding Author's email: longes2@163.com
} 
$\mathrm{W} /\left(\mathrm{m}^{2} \cdot \mathrm{K}\right)$, building heating energy consumption reduced by $12.1 \%$. Jian Zhou, etc. [5] studied the thermal environment and indoor environment performance of the North-East England one office building, points out the outer wall heat transfer coefficient changed from 0.29 $\mathrm{W} /\left(\mathrm{m}^{2} \cdot \mathrm{K}\right)$ to $0.09 \mathrm{~W} /\left(\mathrm{m}^{2} \cdot \mathrm{K}\right)$, building heating energy consumption was reduced by $8.4 \%$.

The temperature difference between indoor and outdoor is also an important factor affecting the energy consumption of building heating. The setting of indoor heating temperature is extremely important when the outdoor climate is certain. Sokratis et al. [6] applied a multi-objective optimization algorithm based on genetic algorithm to large typical office buildings in seven different climatic zones in the United States, and concluded that the heating setting temperature in cold areas was reduced by $0.7^{\circ} \mathrm{C}$, and the building heating system energy saving was $11.53 \%$.Tyler Hoyt et al. [7], on the premise of ensuring thermal comfort, pointed out that in cold areas, the heating temperature setting value was reduced from $21.1^{\circ} \mathrm{C}$ to $20^{\circ} \mathrm{C}$, saving $34 \%$ of energy.

To sum up, most studies at the present stage are about the influence of different enveloping structures or lowering the heating setting temperature on building energy consumption. But the same city has buildings completed at different years, and the Chinese government has long advocated reducing indoor heating temperatures to conserve energy. After a variety of factors are coupled, the research results of different scholars have confirmed the significance of energy saving by reducing the heating set temperature, but the energy saving and energy saving rate differ greatly, so it is difficult to compare with each other. This paper studies the energy saving effect and the microcosmic influencing mechanism of the heating energy consumption of public buildings when the same climate and building parameters are used for the thermal performance of the building envelope at different completion years and the heating setting temperature is reduced by the same range. This paper discusses whether the same city's energy-saving guiding policy of reducing the heating setting temperature by the same range is beneficial to realize the similar energy-saving rate.

\section{Research objects and conditions}

The architectural reference model for this study is a three-storey medium-sized building created by the US Department of Energy (DOE) [8]. The meteorological parameters of Beijing, the representative city in the cold climate zone, are selected as the climatic background of the study. The heating demand is rigid. The building diagram is shown in Figure 1. The height of the building is $4 \mathrm{~m}$, the building area is 4983 square meters, the shape coefficient is 0.22 , the window wall area ratio of each direction is 0.33 .

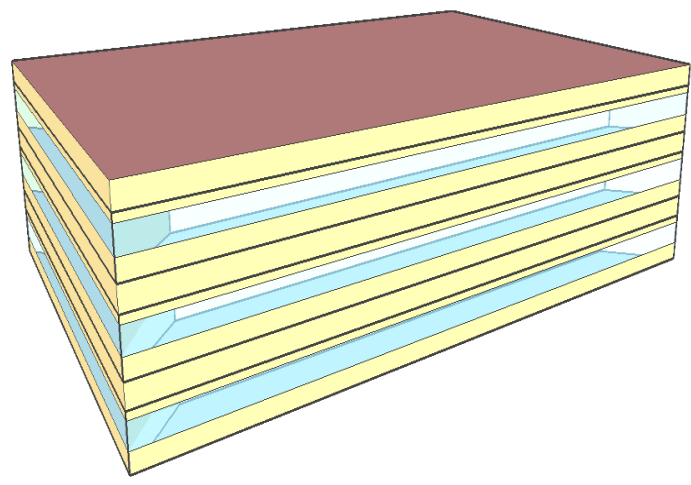

Figure 1. Building schematic Diagram.

In the same city, due to the different functions of the buildings and the different years of completion, the implementation standards of the buildings are also different. According to the regulations of various building standards $[1,2]$ on the thermal performance of enveloping structures, the buildings with different functional uses in Beijing are divided into four periods according to the architectural design period: pre-2005, 2005-2015, 2015-2019 and after 2019. In order to study the difference of energy saving effect of reducing heating setting temperature on buildings of different ages, it is assumed that the building geometric parameters are exactly the same. According to the age of architectural design, the envelope is divided into four thermal properties: Poor, Medium, Good and Excellent. With the overall improvement of the thermal performance of envelope, the thermal parameters of envelope decrease. The specific indexes of thermal performance combination of envelope structure are shown in Table 1.

Table 1. Combination of thermal performance of envelope

\begin{tabular}{|c|c|c|c|c|c|c|}
\hline \multirow{2}{*}{$\begin{array}{c}\text { Completion } \\
\text { years }\end{array}$} & $\begin{array}{c}\text { Thermal } \\
\text { performance of } \\
\text { envelope }\end{array}$ & $\begin{array}{c}\text { K(Exterior } \\
\text { wall) }\end{array}$ & $\begin{array}{c}\text { K (Interior } \\
\text { wall) }\end{array}$ & K (Window) & $\mathrm{K}$ (Roof) & $\mathrm{K}$ (Floor) \\
\cline { 3 - 8 } & Poor & 1.4 & 2.33 & 4.5 & 1 & 2.33 \\
\hline Pre-2005 & Medium & 1 & 2.03 & 3.5 & 0.6 & 2.03 \\
\hline $2005-2015$ & Good & 0.6 & 1.83 & 2.5 & 0.3 & 1.83 \\
\hline After 2015-2019 & Excellent & 0.2 & 1.63 & 1 & 0.15 & 1.62 \\
\hline
\end{tabular}

The initial heating setting temperature is $18^{\circ} \mathrm{C}$, and only $1^{\circ} \mathrm{C}\left(17^{\circ} \mathrm{C}\right)$ is lowered to study its rule. This paper simulates and predicts the hourly dynamic heat load of the thermal performance of four enveloping structures before and after the heating set temperature is reduced, calculates the energy saving and energy saving ratio when the heating set temperature is reduced by $1^{\circ} \mathrm{C}$ throughout the year, and reveals the micro-level mechanism with common and individual rules. The 
simulation method adopts the characteristic temperature method (CTM method for short) [9]. Based on variable control, the energy-saving effect of reducing heating temperature by the same extent in buildings in different completion years was emphasized, and the different functional uses of buildings were uniformly treated. In this study, the internal heat source was not considered for the time being. The work and rest time was set at 9:00 17:00, and the number of indoor fresh air ventilation was set at $0.51 / \mathrm{h}$. The shading coefficient of the enclosure window is set as 1 , and the solar radiation transmittance is set as 0.8 .

\section{Annual energy saving effect}

Figure 2 shows the comparison of annual energy saving and energy saving ratio when the set heating temperature of buildings decreases by $1^{\circ} \mathrm{C}$ in different years. It can be seen from the figure that with the improvement of the thermal performance of the envelope, the annual energy saving when the heating temperature is reduced by $1{ }^{\circ} \mathrm{C}$ is significantly reduced. This indicates that for buildings with a long completion age and poor thermal performance of the envelope, the greater the annual energy saving when the heating temperature is reduced by $1{ }^{\circ} \mathrm{C}$ is, the more significant the energy saving effect is. With the improvement of the thermal performance of the envelope, the annual energy saving ratio of heating temperature decreasing by $1{ }^{\circ} \mathrm{C}$ slightly decreases, indicating that the annual energy saving ratio of heating temperature decreasing by $1{ }^{\circ} \mathrm{C}$ is similar regardless of the age of the building completion and the thermal performance of the envelope.

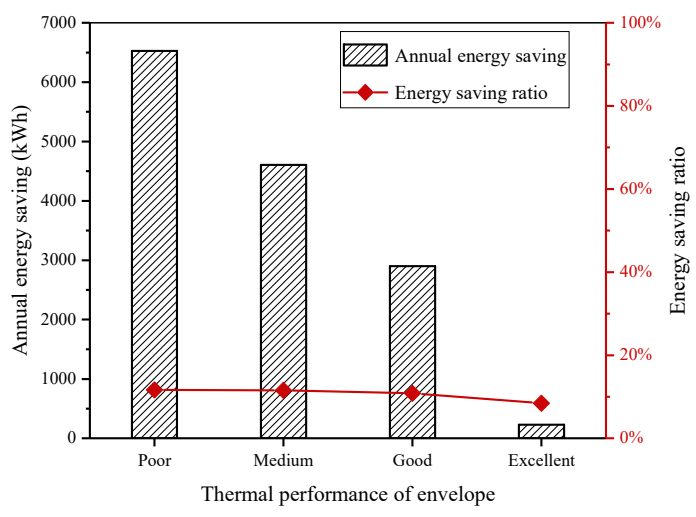

Figure 2. Comparison of energy saving effects throughout the year.

The above results show that lowering the heating setting temperature can reduce heating energy consumption for buildings of different completion ages. Although the difference of energy saving was significant, the relative energy saving ratio was basically similar without considering the difference of internal heat source in residential behavior. Justifies China's building energy efficiency standard system or guidance policy on setting temperature to achieve similar energy saving ratio targets. In addition, for the old buildings with the worse thermal performance of the envelope, lowering the heating setting temperature can not only achieve the goal of relative energy saving ratio, but also achieve more significant absolute energy saving effect and reduce the heating expense more obviously in the economic aspect.

\section{Time-by-moment energy saving mechanism}

Figure 3 shows the variation rule of hourly load reduction with thermal load in buildings of different ages. It can be seen in the same envelope thermal properties, along with the rising of the hourly thermal load, hourly load reduction increases first along the slope of 1 line, and then basically remains unchanged. Moreover, the stability value of hourly load reduction varies significantly under different thermal performance of envelope, specifically, the older building with worse thermal performance of envelope decreases more hourly load.

For different enveloping structures, the worse the thermal performance of enveloping structures is when there is heating demand before and after the heating set temperature is reduced, the greater the hourly load reduction of the same temperature reduction range is, and the difference of hourly load reduction is obvious under the different enveloping structures' thermal performance.

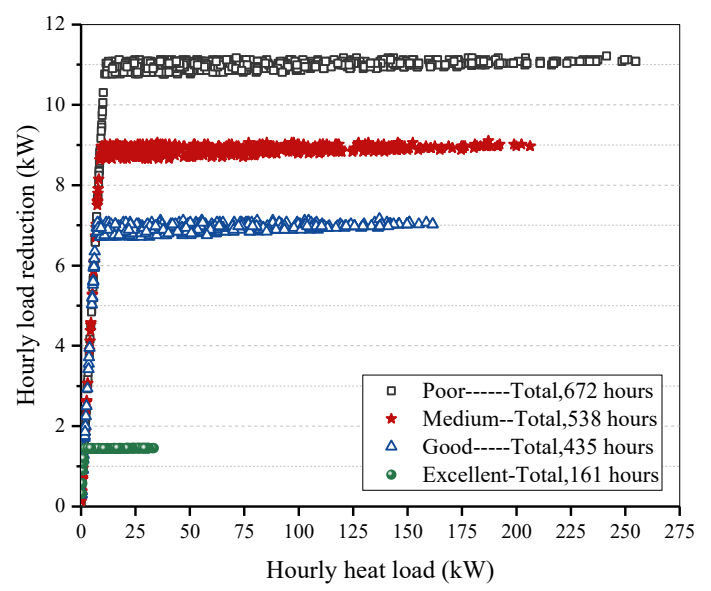

Figure 3. The change rule of hourly load reduction with thermal load

Figure 4 shows the change rule of hourly load reduction ratio of buildings with hourly thermal load in different ages. Figure (a) is the abscissa of the hourly thermal load, figure (b) the hourly dimensionless thermal load. The abscissa is the ratio of hourly thermal load and corresponding maximum hourly thermal load under each envelope structure. The range of hourly thermal load variation of different enclosure structures is uniformly set as $0 \sim 100 \%$, so as to compare the change of hourly load reduction ratio more intuitively.

It can be seen from Figure (a) that under the same building with the same envelope structure, with the increase of hourly heat load, the hourly load reduction rate is at first $100 \%$, then rapidly decreases along the inverse proportion function, and finally tends to a similar stable value. 
According to figure (b), it can be seen intuitively. After the hour-by-hour heat load is dimensioned under the thermal performance of each envelope, the hour-byhour load reduction ratio curves of the heating temperature reduced by $1^{\circ} \mathrm{C}$ of different envelope thermal performance are highly consistent, indicating that the hour-by-hour load reduction ratio of the heating temperature reduced by $1^{\circ} \mathrm{C}$ of different envelope thermal performance has little difference.

Based on the comprehensive figure (a) and figure (b), it can be seen that the hourly load reduction ratio is similar regardless of the performance of the envelope structure. This also analyzes the law of similar annual energy saving ratio from the perspective of microscopic mechanism, proving that for buildings in different completion years, similar energy saving ratio can be realized by reducing the heating setting temperature by the same extent.
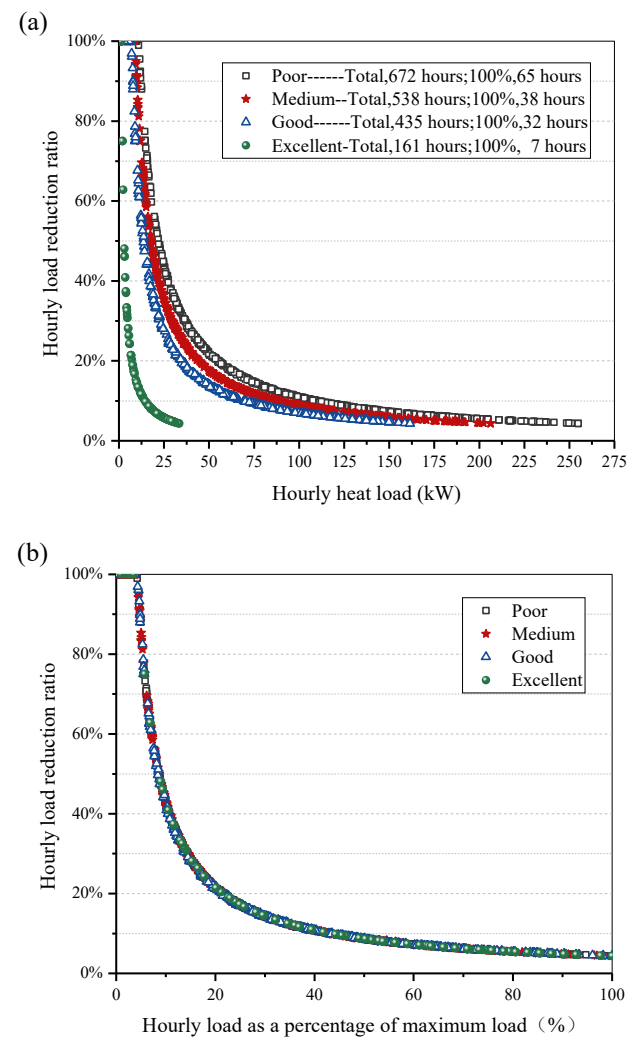

Figure 4. The change rule of hourly load reduction ratio with thermal load

\section{Conclusion}

This paper takes the same building in the same city as the research object. Under the assumption of four different grade envelopment structures, the annual macro energy saving effect of four envelopment structures representing different completion years was studied when the heating setting temperature was reduced by $1^{\circ} \mathrm{C}$. The mechanism of energy saving at the micro-level is deeply analyzed, and then the annual energy saving effect of different building functions and uses is discussed. The conclusions are as follows:
(1) The annual energy saving difference of the heating set temperature with the same reduction is significant under different thermal performance of the envelope. The worse the thermal performance of the envelope, the greater the annual heat consumption, and the greater the annual energy saving when the heating setting temperature decreases by the same extent. It indicates that the older the building is, the better heating energy saving effect and more heating cost can be achieved by advocating lowering the heating setting temperature of the same range.

(2)There is little difference in annual energy saving ratio when the heating set temperature is reduced by the same range under different thermal performance of envelope. In addition, the annual energy saving ratio of the old buildings with the worse enclosure structure is slightly higher, and that of the new buildings with the better enclosure structure is slightly lower, but generally similar. It shows that it is reasonable for the same city to advocate the policy of reducing heating temperature by the same range to achieve the goal of similar energy saving ratio.

\section{References}

1. Energy Conservation design standards for public Buildings GB 50189-2015.

2. Technical standards for near-zero energy buildings GB/T 51350-2019.

3. General Office of the State Council. Notice of The General Office of the State Council on strict implementation of air Conditioning Temperature Control Standards for Public Buildings [EB/OL] .

4. N. Casquero-Modrego, M. Goñi-Modrego. Energy retrofit of an existing affordable building envelope in Spain, case study [J]. Sustainable Cities and Society,44 (2019), 395-405.

5. J. Zhou, W. Nazi, Y. Wang, A. Roskilly. Investigating the impact of building's facade on the building's energy performance-a case study [J]. Energy Procedia, 158 (2019) :3144-3151.

6. S. Papadopoulos, C. Kontokosta, A. Vlachokostas, E. Azar. Rethinking HVAC temperature setpoints in commercial buildings: The potential for zero-cost energy savings and comfort improvement in different [J]. Building and Environment ,155 (2019), 350-359.

7. T. Hoyt, E. Arens, H. Zhang. Extending air temperature setpoints: Simulated energy savings and design considerations for new and retrofit buildings [J]. Building and Environment, 88 (2015),89-96.

8. Deru M, Field K, Studer D, Benne K, Griffith B, Torcellini P, et al. Department of Energy Commercial reference building models of national building stock. 2011. NREL Report TP-5500e46861.

9. E. Long. Genetic Theory of building Energy Consumption and Practice of Building Energy Conservation [M]. Beijing: Science Press, 2009. 